\title{
Two families of Entanglement-assisted quantum MDS codes from constacyclic codes
}

\author{
Liangdong $\mathrm{Lu}^{a, b, \dagger}$, Wenping $\mathrm{Ma}^{a}$, Luobin $\mathrm{Guo}^{b}$ \\ a. State Key Laboratory of Integrated Services Networks, Xidian University, \\ Xi'an, Shaanxi, 710051, China, (email: † kelinglv@163.com, ) \\ b. Department of Basic Science, Air Force Engineering University, Xi'an, Shaanxi, \\ 710051, China
}

\begin{abstract}
Entanglement-assisted quantum error correcting codes (EAQECCs) can be derived from arbitrary classical linear codes. However, it is a very difficult task to determine the number of entangled states required. In this work, using the method of the decomposition of the defining set of constacyclic codes, we construct two families of $q$-ary entanglement-assisted quantum MDS (EAQMDS) codes based on classical constacyclic MDS codes by exploiting less pre-shared maximally entangled states. We show that a class of $q$-ary EAQMDS have minimum distance upper bound greater than $q$. Some of them have much larger minimum distance than the known quantum MDS (QMDS) codes of the same length. Most of these $q$-ary EAQMDS codes are new in the sense that their parameters are not covered by the codes available in the literature.
\end{abstract}

Index terms: Entanglement-assisted quantum error correcting codes (EAQECCs), MDS codes, cyclotomic cosets, constacyclic code.

\section{Introduction}

Since the significant discovery was published in [1, 2], the theory of quantum errorcorrecting codes has experienced tremendous grouth. The most widely studied construction of quantum error-correcting codes is the stabilizer formalism [3, 5]. It allows standard quantum codes to be constructed from dual-containing (or self-orthogonal) classical 
codes [3]. However, the dual-containing condition forms a barrier in the development of quantum coding theory. In Ref. 4], Brun et al. proposed a entanglement-assisted (EA) stabilizer formalism which shown that non-dual-containing classical codes can be used to construct EAQECCs if shared entanglement is available between the sender and receiver.

Let $q$ be a prime power. A $q$-ary $[[n, k, d ; c]]$ EAQECC that encodes $k$ information qubits into $n$ channel qubits with the help of $c$ pairs of maximally-entangled Bell states can correct up to $\left\lfloor\frac{d-1}{2}\right\rfloor$ errors, where $d$ is the minimum distance of the code. A $q$-ary $[[n, k, d ; c]]$ EAQECC is denoted by $[[n, k, d ; c]]_{q}$. As the same as in classical coding theory, one of the fundamental tasks in quantum coding theory is to construct quantum codes with the best possible minimum distance. For EA-quantum codes, one of the most known bound is EA-Quantum Singleton Bound as follow.

Theory $1.1\left[4,8\right.$. ( EA-Quantum Singleton Bound) An $[[n, k, d ; c]]_{q}$ EAQECC satisfies

$$
n+c-k \geq 2(d-1)
$$

where $0 \leq c \leq n-1$.

A code for which equality holds in the EA-Quantum Singleton Bound is called EAquantum maximum-distance-separable (EA-QMDS). If $c=0$, then this bound is Quantum Singleton Bound, and a code for which equality holds in the bound is called quantum maximum-distance-separable (QMDS). Both QMDS codes and EAQMDS codes are an important type of quantum codes. Therefore, constructing QMDS codes and EAQMDS codes have became a central topic for quantum error-correction codes. Currently, many QMDS codes have been constructed by different methods [17 25]. In [5], the MDS conjecture shown that the length of maximal-distance-separable (MDS) code cannot exceed $q^{2}+1$. It is shown that except for some spares lengths $n$, all known $q$-ary quantum MDS codes have minimum distance less than or equal to $\frac{q+1}{2}$. It is a very difficult task to construct quantum MDS codes with relatively large minimum distance. In order to construct some quantum MDS codes with larger minimal distance, many scholars refer to construct EA-quantum MDS codes that have larger minimal distances than the quantum codes with the same length $n$. One of the most frequently used construction methods is as follows.

Proposition 1.2 [4,7]. Let $\mathcal{C}=[n, k, d]_{q^{2}}$ be a classical code over $F_{q^{2}}$ with parity check matrix $H$. There exists an $[[n, 2 k-n+c, d ; c]]_{q}$ EAQECC, where $c=\operatorname{rank}\left(H H^{\dagger}\right)$ is the number of maximally entangled states required and $H^{\dagger}$ is the conjugate matrix of $H$ over 
$F_{q^{2}}$.

In resent years, lots of scholars have constructed many entanglement-assisted quantum codes with good parameters, see [4,6-11, 13, 16, 31, 41] . In [35], we proposed the concept about a decomposition of the defining set of constacyclic codes. With the help of this concept, we construct some good entanglement-assisted quantum MDS codes. In order to discover characters of concept and its applications, in this paper, we continue to construct two families of EA-quantum MDS codes with length $n$ from classical constacyclic codes by the decomposition of the defining set. In [18, it is shown that dual-containing constacylic codes over $F_{q^{2}}$ exist only if the order $r$ is a divisor of $q+1$. So, We pay attention to constacyclic codes with $r \mid q+1$ that can be used to construct EA-quantum MDS codes. More precisely, Our main contribution on new $q$-ary quantum MDS codes is as follows:

(1) $\left[\left[\frac{q^{2}-1}{2 h}, \frac{q^{2}-1}{2 h}-2 d+3, d ; 1\right]\right]_{q}$, where $q$ is an odd prime power, $h \in\{3,5,7\}, 2 h \mid q+1$ and $\frac{q+1}{h}+1 \leq d \leq \frac{(q+1)(h+3)}{2 h}-1$.

(2) $[[2 \lambda(q-1), 2 \lambda(q-1)-2 d+2 i, d ; 2 i]]_{q}$, where $q$ be an odd prime power with $8 \mid q+1$, $\lambda$ is an odd divisor of $q+1$ and $\frac{q-1}{2}(i-1)+4 \lambda+1 \leq d \leq \frac{q-1}{2}+2(i+1) \lambda$;

In construction (1), consumed only one pair of maximally entangled states, some EAquantum MDS codes with the minimal distance upper limit larger than $q$ are obtained.In construction (2), consumed two pairs of maximally entangled states, some of EA-quantum MDS codes with the minimal distance are larger than the standard quantum MDS codes in Ref. [18. Consumed four pairs of maximally entangled states, most of all EA-quantum MDS codes with the minimal distance are much larger than $q$.

The paper is organized as follows. In Section 2, basic results about constacyclic codes and EA-quantum codes are provided. The concept of a decomposition of the defining set of constacyclic codes is stated. In Section 3, we give some new classes of EA-quantum MDS codes. The conclusion is given in Section 4 .

\section{Preliminaries}

In this section, we review some basic results on constacyclic codes, $\mathrm{BCH}$ codes, and EAQECCs for the purpose of this paper. For details on BCH codes and constacyclic codes can be found in standard textbook on coding theory [28, 29], and for EAQECCs please see Refs. [4,6-15]. 
Let $p$ be a prime number and $q$ a power of $p$, ie., $q=p^{l}$ for some $l>0$. We denotes the finite field with $q^{2}$ elements as $F_{q^{2}}$. Given any $\alpha \in F_{q^{2}}$, the conjugation of $\alpha$ is denoted by $\bar{\alpha}=\alpha^{q}$. For two vectors $\mathbf{x}=\left(x_{1}, x_{2}, \cdots, x_{n}\right)$ and $\mathbf{y}=\left(y_{1}, y_{2}, \cdots, y_{n}\right) \in F_{q^{2}}^{n}$, the Hermitian inner product is defined as $(\mathbf{x}, \mathbf{y})_{h}=\sum \overline{x_{i}} y_{i}=\overline{x_{1}} y_{1}+\overline{x_{2}} y_{2}+\cdots+\overline{x_{n}} y_{n}$. For a linear code $\mathcal{C}$ over $F_{q^{2}}$ of length $n$, the Hermitian dual code $\mathcal{C}^{\perp_{h}}$ is defined as $\mathcal{C}^{\perp_{h}}=\left\{x \in F_{q^{2}}^{n} \mid(x, y)_{h}=0, \forall y \in \mathcal{C}\right\}$. If $\mathcal{C}^{\perp_{h}} \subseteq \mathcal{C}$, then $\mathcal{C}$ is called a Hermitian dual containing code, and $\mathcal{C}^{\perp_{h}}$ is called a Hermitian self-orthogonal code.

We now recall some results about cyclic codes and classical constacyclic codes. For any vector $\left(c_{0}, c_{1}, \cdots, c_{n-1}\right) \in F_{q^{2}}^{n}$, a $q^{2}$-ary linear code $\mathcal{C}$ of length $n$ is called $\eta$-constacyclic if it is invariant under the $\eta$-constacyclic shift of $F_{q^{2}}^{n}$ :

$$
\left(c_{0}, c_{1}, \cdots, c_{n-1}\right) \rightarrow\left(\eta c_{n-1}, c_{0}, \cdots, c_{n-2}\right),
$$

where $\eta$ is a nonzero element of $F_{q^{2}}$. Moreover, $\mathcal{C}$ is called a cyclic code if $\eta=1$; and $\mathcal{C}$ is called a negacyclic code if $\eta=-1$.

For a constacyclic code $\mathcal{C}$, each codeword $c=\left(c_{0}, c_{1}, \cdots, c_{n-1}\right)$ is customarily represented in its polynomial form: $c(x)=c_{0}+c_{1} x+\cdots+c_{n-1} x_{n-1}$, and the code $\mathcal{C}$ is in turn identified with the set of all polynomial representations of its codewords. For studying constacyclic codes, the proper content is the residue class $\operatorname{ring} \mathcal{R}_{n}=\mathbb{F}_{q}[x] /\left(x^{n}-\eta\right) . x c(x)$ corresponds to a constacyclic shift of $c(x)$ in the $\operatorname{ring} \mathcal{R}_{n}$. As well known, a linear code $\mathcal{C}$ of length $n$ over $F_{q^{2}}$ is constacyclic if and only if $\mathrm{C}$ is an ideal of the quotient ring $\mathcal{R}_{n}=\mathbb{F}_{q}[x] /\left(x^{n}-\eta\right)$. It follows that $\mathcal{C}$ is generated by monic factors of $\left(x^{n}-\eta\right)$, i.e., $\mathcal{C}=\langle f(x)\rangle$ and $f(x) \mid\left(x^{n}-\eta\right)$. The $f(x)$ is called the generator polynomial of $\mathcal{C}_{n}$.

Let $\eta \in F_{q^{2}}$ be a primitive $r$ th root of unity. Let $\operatorname{gcd}(n, q)=1$, then there exists a primitive $r n$-th root of unity $\omega$ in some extension field field of $F_{q^{2}}$ such that $\omega^{n}=\eta$. Hence, $x^{n}-\eta=\prod_{i=0}^{n-1}\left(x-\omega^{1+i r}\right)$. Let $\Omega=\{1+i r \mid 0 \leq i \leq n-1\}$. For each $j \in \Omega$, let $C_{j}$ be the $q^{2}$-cyclotomic coset modulo $r n$ containing $j$. Let $\mathcal{C}$ be an $\eta$-constacyclic code of length $n$ over $F_{q^{2}}$ with generator polynomial $g(x)$. The set $T=\left\{j \in \Omega \mid g\left(\omega^{j}\right)=0\right\}$ is called the defining set of $\mathcal{C}$. Let $s$ be an integer with $0 \leq s<r n$, the $q^{2}$-cyclotomic coset modulo $r n$ that contains $s$ is defined by the set $C_{s}=\left\{s, s q^{2}, s q^{2 \cdot 2}, \cdots, s q^{2(k-1)}\right\}(\bmod r n)$, where $k$ is the smallest positive integer such that $x q^{2 k} \equiv x(\bmod r n)$. We can see that the defining set $T$ is a union of some $q^{2}$-cyclotomic cosets module $r n$ and $\operatorname{dim}(\mathcal{C})=n-|T|$.

Let $\mathcal{C}$ be a constacyclic code with a defining set $T=\bigcup_{s \in S} C_{s}$. Denoted $T^{-q}=\{r n-$ 
$q s \mid s \in T\}$, then the defining set of $\mathcal{C}^{\perp_{h}}$ is $T^{\perp_{h}}=\Omega \backslash T^{-q}$ [23]. Since there is a striking similarity between cyclic codes and constacyclic code, we give a correspondence defining of skew aymmetric and skew asymmetric as follows. A cyclotomic coset $C_{s}$ is skew symmetric if $r n-q s \bmod r n \in C_{s}$; and otherwise is skew asymmetric otherwise. Skew asymmetric cosets $C_{s}$ and $C_{r n-q s}$ come in pair, we use $\left(C_{s}, C_{r n-q s}\right)$ to denote such a pair.

The following results on $q^{2}$-cyclotomic cosets, dual containing constacyclic codes are bases of our discussion.

Lemma 2.1 [23, 27]. Let $r$ be a positive divisor of $q+1$ and $\eta \in F_{q^{2}}^{*}$ be of order $r$. Let $\mathcal{C}$ be a $\eta$-constacyclic code of length $n$ over $F_{q^{2}}$ with defining set $T$, then $\mathcal{C}^{\perp_{h}} \subseteq \mathcal{C}$ if and only if one of the following holds:

(1) $T \cap T^{-q}=\emptyset$, where $T^{-q}=\{r n-q s \mid s \in T\}$.

(2) If $i, j, k \in T$, then $C_{i}$ is not a skew asymmetric coset and $\left(C_{j}, C_{k}\right)$ is not a skew asymmetric cosets pair.

From Lemma $2.1, \mathcal{C}^{\perp_{h}} \subseteq \mathcal{C}$ can be described by the relationship of its cyclotomic coset $C_{s}$. However, a defining set $T$ of a non-dual-containing (or non-self-orthogonal) classical codes is $T \cap T^{-q} \neq \emptyset$. In order to construct EA-quantum MDS codes for larger distance than $q+1$ of code length $n \leq q^{2}+1$, we recall the fundamental definition of decomposition of the defining set of constacyclic codes [35]. There are also other types of definition for decomposition of the defining set of cyclic codes, negacyclic codes, see [14, 26, 33, 34].

Definition 2.2 [35] Let $\eta \in F_{q^{2}}$ be a primitive rth root of unity. Let $\mathcal{C}$ be a $\eta$ constacyclic code of length $n$ with defining set $T$. Denote $T_{s s}=T \cap T^{-q}$ and $T_{\text {sas }}=T \backslash T_{s s}$, where $T^{-q}=\{r n-q x \mid x \in T\}$ and $r$ is a factor of $q+1 . T=T_{s s} \cup T_{\text {sas }}$ is called decomposition of the defining set of $\mathcal{C}$.

To determine $T_{s s}$ and $T_{\text {sas }}$, we give the following lemma to characterize them.

Lemma 2.3 [27]. Let $\operatorname{gcd}(q, n)=1, \operatorname{ord}_{r n}\left(q^{2}\right)=m, 0 \leq x, y, z \leq n-1$.

(1) $C_{x}$ is skew symmetric if and only if there is a $t \leq\left\lfloor\frac{m}{2}\right\rfloor$ such that $x \equiv x q^{2 t+1}(\bmod$ n).

(2) If $C_{y} \neq C_{z},\left(C_{y}, C_{z}\right)$ form a skew asymmetric pair if and only if there is a $t \leq\left\lfloor\frac{m}{2}\right\rfloor$ such that $y \equiv z q^{2 t+1}(\bmod \mathrm{n})$ or $z \equiv y q^{2 t+1}(\bmod \mathrm{n})$.

Using the decomposition of a defining set $T$, one can calculate the number of needed ebits with a algebra method.

Lemma 2.4 [35]. Let $T$ be a defining set of a constacyclic code $\mathcal{C}, T=T_{s s} \cup T_{\text {sas }}$ be 
decomposition of $T$. Using $\mathcal{C}^{\perp_{h}}$ as EA stabilizer, the optimal number of needed ebits is $c=\left|T_{s s}\right|$.

Lemma 2.5 [30]. (The BCH bound for Constacyclic Codes) Let $\mathcal{C}$ be an $\eta$-constacyclic code of length $n$ over $F_{q^{2}}$, where $\eta$ is a primitive $r$ th root of unity. Let $\omega$ be a primitive $r n$-th root of unity in an extension field of $F_{q^{2}}$ such that $\omega^{n}=\eta$. Assume the generator polynomial of $\mathcal{C}$ has roots that include the set $\left\{\omega^{1+r i} \mid i_{1} \leq i \leq i_{1}+d-2\right\}$. Then the minimum distance of $\mathcal{C}$ is at least $d$.

Lemma $2.6[4,14]$ Let $\mathcal{C}$ be an $[n, k, d]_{q^{2}}$ constacyclic code with defining set $T$, and the decomposition of $T$ be $T=T_{s s} \cup T_{\text {sas }}$. Then $\mathcal{C}^{\perp_{h}}$ EA stabilizes an $q$-ary $[[n, n-2|T|+$ $\left.\left.\left|T_{s s}\right|, d \geq \delta ;\left|T_{s s}\right|\right]\right]$ EAQECC.

\section{New EA-quantum MDS Codes}

In this section, we consider $\eta$-constacyclic codes over $F_{q^{2}}$ of length $n$ to construct EAquantum codes. To do this, we give a sufficient condition for a decomposition of the defining set of $\eta$-constacyclic codes over $F_{q^{2}}$ of length $n$ which do not contain their Hermitian duals. First, we compute $q^{2}$-cyclotomic cosets modulo $r n$ where $r \mid q+1$ (constacyclic codes).

\subsection{Lenght $n=\frac{q^{2}-1}{2 h}$ with $h \in\{3,5,7\}$}

Let $h \in\{3,5,7\}, q$ be an odd prime power with $2 h \mid(q+1)$. Suppose $n=\frac{q^{2}-1}{2 h}$ and $r=h$. Let $\eta \in F_{q^{2}}$ be a primitive $r^{\text {th }}$ root of unity. Since $r n \mid q^{2}-1$ clearly, every $q^{2}$-cyclotomic coset modulo $r n$ contains exactly one element.

In this subsection, adding one ebit, we construct a new family of a family of new EAquantum MDS codes with parameters $\left[\left[\frac{q^{2}-1}{2 h}, \frac{q^{2}-1}{2 h}-2 d+3, d ; 1\right]\right]$, where $\frac{q+1}{2 h}+1 \leq d \leq$ $\frac{(h+1)(q+1)}{2 h}-1$.

Lemma 3.1: Let $q$ is an odd prime power with $2 h \mid(q+1), h \in\{3,5,7\}$ and $n=\frac{q^{2}-1}{2 h}$. If $\mathcal{C}$ is a $q^{2}$-ary constacyclic code of length $n$ with define set $T=\bigcup_{i=\frac{(h-2)(q+1)}{2 h}}^{k}\left\{C_{1+h i}\right\}$, where $\frac{(h-2)(q+1)}{2 h} \leq k \leq \frac{(2 h-1)(q+1)}{2 h}-3$, and the decomposition of a defining set $T=T_{s s} \cup T_{\text {sas }}$, then

(i) $C_{1+\left(\frac{(h-1)(q+1)}{2 h}-1\right) h}$ is skew symmetric. $\quad$ (ii) $\left|T_{s s}\right|=1$, if $\frac{(h-1)(q+1)}{2 h}-1 \leq k \leq$ $\frac{(2 h-1)(q+1)}{2 h}-3$. 
Proof: (i) Since $1+\left(\frac{(h-1)(q+1)}{2 h}-1\right) h=\frac{(h-1)(q-1)}{2}$ and $-\left[1+\left(\frac{(h-1)(q+1)}{2 h}-1\right) h\right] q \equiv$ $-\frac{(h-1)(q-1)}{2} q \equiv-\left[\frac{h-1}{2}\left(q^{2}-1\right)-\frac{(h-1)(q-1)}{2}\right] \equiv \frac{(h-1)(q-1)}{2} \equiv 1+\left(\frac{(h-1)(q+1)}{2 h}-1\right) h \bmod h n$, $C_{1+\left(\frac{(h-1)(q+1)}{2 h}-1\right) h}$ is skew symmetric.

(ii) Let $T=\bigcup_{i=\frac{(h-3)(q+1)}{2 h}}^{k}\left\{C_{1+h i}\right\}$, where $\frac{(h-3)(q+1)}{2 h} \leq k \leq q-2$. Since $C_{1+\left(\frac{(h-1)(q+1)}{2 h}-1\right) h}$ is skew symmetric, $T_{s s}$ comprises the set $\left\{C_{1+\left(\frac{(h-1)(q+1)}{2 h}-1\right) h}\right\}$ at least. According to the concept about a decomposition of the defining set $T$, one obtain that $T_{s a s}=T \backslash T_{s s}$. In order to testify $\left|T_{s s}\right|=1$ if $\frac{(h-1)(q+1)}{2 h}-1 \leq i \leq q-2$, from Definition 2.2 and Lemma 2.3 , we need to testify that there is no skew symmetric cyclotomic coset, and any two cyclotomic coset do not form a skew asymmetric pair in $T_{\text {sas }}$.

Let $I=\left\{1+h i \mid \frac{(h-3)(q+1)}{2 h} \leq i \leq q-2\right\} \backslash\left(1+\left(\frac{(h-1)(q+1)}{2 h}-1\right) h\right)$ and $r=h$. Only we need to testy that for $\forall x \in I,-q x(\bmod r n) \notin I$ and $T_{s s}=\left\{C_{1+\left(\frac{(h-1)(q+1)}{2 h}-1\right) h}\right\}$. That implies that if $x, y \in I, C_{x}$ is not a skew symmetric cyclotomic coset, and any $C_{x}, C_{y}$ do not form a skew asymmetric pair if and only if $x+y q \not \equiv 0 \bmod r n$.

Divide $I$ into three parts $I_{1}=\left[1+\frac{(h-3)(q+1)}{2 h} h, 1+\left(\frac{(h-1)(q+1)}{2 h}-2\right) h\right], I_{2}=[1+$ $\left.\left(\frac{(h-1)(q+1)}{2 h}\right) h, 1+\left(\frac{(h+1)(q+1)}{2 h}-2\right) h\right]$ and $I_{3}=\left[1+\left(\frac{(h+1)(q+1)}{2 h}-1\right) h, 1+(q-2) h\right] . \quad r n=$ $h n=q^{2}-1$. If $x, y \in I_{1}$, then $\frac{(h-3)}{2}\left(q^{2}-1\right)+(h-2)(q+1)=\left(1+\frac{(h-3)(q+1)}{2 h} h\right)(q+1) \leq$ $x+y q \leq\left(1+\left(\frac{(h-1)(q+1)}{2 h}-2\right) h\right)(q+1)=\frac{(h-1)}{2}\left(q^{2}-1\right)-h(q+1)$. Since $h \in\{3,5,7\}$, $\frac{(h-3)}{2}=\{0,1,2\}$, and $\frac{(h-1)}{2}=\{1,2,3\}$. Hence, for $x, y \in I_{1}, l n<x+y q<(l+1) n$, where $0 \leq l \leq 2$ is a integer.

If $x, y \in I_{2}$, then $\frac{h-1}{2}\left(q^{2}-1\right)+h(q+1)=\left(1+\left(\frac{(h-1)(q+1)}{2 h}\right) h\right)(q+1) \leq x+y q \leq(1+$ $\left.\left(\frac{(h+1)(q+1)}{2 h}-2\right) h\right)(q+1)=\frac{h+1}{2}\left(q^{2}-1\right)-(h-2)(q+1)$. Since $h \in\{3,5,7\}, \frac{(h-1)}{2}=\{1,2,3\}$, and $\frac{(h+1)}{2}=\{2,3,4\}$. Hence, for $x, y \in I_{2}, l n<x+y q<(l+1) n$, where $1 \leq l \leq 3$ is a integer.

For $h=3$, if $x, y \in I_{3}$, then $2 n<\frac{h+1}{2}\left(q^{2}-1\right)+2(q+1)=\left(1+\left(\frac{(h+1)(q+1)}{2 h}-1\right) h\right)(q+1) \leq$ $x+y q \leq(1+(q-2) h)(q+1)=h\left(q^{2}-1\right)-(h-1)(q+1)<3 n$.

For $h=5$, Divide $I_{3}$ into two parts $I_{3}^{\prime}=\left[1+\left(\frac{(h+1)(q+1)}{2 h}-1\right) h, 1+\left(\frac{(h+3)(q+1)}{2 h}-2\right) h\right]$ and $I_{3}^{\prime \prime}=\left[1+\left(\frac{(h+3)(q+1)}{2 h}-1\right) h, 1+(q-2) h\right]$.

if $x, y \in I_{3}^{\prime}$, then $3 n<\frac{h+1}{2}\left(q^{2}-1\right)+2(q+1)=\left(1+\left(\frac{(h+1)(q+1)}{2 h}-1\right) h\right)(q+1) \leq x+y q \leq$ $\left(1+\left(\frac{(h+3)(q+1)}{2 h}-2\right) h\right)(q+1)=\frac{h+3}{2}\left(q^{2}-1\right)-(h-2)(q+1)<4 n$; if $x, y \in I_{3}^{\prime \prime}, 4 n<$ $\left(1+\left(\frac{(h+3)(q+1)}{2 h}-2\right) h\right)(q+1) \leq x+y q \leq(1+(q-2) h)(q+1)=h\left(q^{2}-1\right)-(h-1)(q+1)<5 n$.

For $h=7$, Divide $I_{3}$ into two parts $I_{3}^{a}=\left[1+\left(\frac{(h+1)(q+1)}{2 h}-1\right) h, 1+\left(\frac{(h+3)(q+1)}{2 h}-2\right) h\right]$, $I_{3}^{b}=\left[1+\left(\frac{(h+3)(q+1)}{2 h}-1\right) h, 1+\left(\frac{(h+5)(q+1)}{2 h}-2\right) h\right]$ and $I_{3}^{c}=\left[1+\left(\frac{(h+5)(q+1)}{2 h}-1\right) h, 1+(q-2) h\right]$. 
If $\left.x, y \in I_{3}^{a}, 4 n<\frac{h+1}{2}\left(q^{2}-1\right)+2(q+1)=\left(1+\left(\frac{(h+1)(q+1)}{2 h}-1\right) h\right) h\right)(q+1) \leq x+$ $y q \leq\left(1+\left(\frac{(h+3)(q+1)}{2 h}-2\right) h\right)(q+1)=\frac{h+3}{2}\left(q^{2}-1\right)-(h-2)(q+1)<5 n$; if $x, y \in I_{3}^{b}$, $5 n<\left(1+\left(\frac{(h+3)(q+1)}{2 h}-2\right) h\right)(q+1) \leq x+y q \leq\left(1+\left(\frac{(h+5)(q+1)}{2 h}-2\right) h\right)(q+1)=\frac{h+5}{2}\left(q^{2}-\right.$ $1)-(h-2)(q+1)<6 n$. if $x, y \in I_{3}^{c}, 6 n<\left(1+\left(\frac{(h+3)(q+1)}{2 h}-1\right) h\right)(q+1) \leq x+y q \leq$ $(1+(q-2) h)(q+1)=h\left(q^{2}-1\right)-(h-1)(q+1)<7 n$.

Hence, there is no skew symmetric cyclotomic cosets, and any two cyclotomic coset do not form a skew asymmetric pair in $T \backslash\left\{C_{1+\left(\frac{(h-1)(q+1)}{2 h}-1\right) h}\right\}$. That implies that $T_{s s}=\left\{C_{1+\left(\frac{(h-1)(q+1)}{2 h}-1\right) h}\right\}$ and $\left|T_{s s}\right|=1$ for $\frac{(h-1)(q+1)}{2 h}-1 \leq i \leq q-2$, when the defining set $T=\bigcup_{i=\frac{(h-3)(q+1)}{2 h}}^{k}\left\{C_{1+h i}\right\}$, where $\frac{(h-3)(q+1)}{2 h} \leq k \leq q-2$.

Theory 3.2: Let $q$ is an odd prime power with $2 h \mid(q+1), h \in\{3,5,7\}$ and $n=\frac{q^{2}-1}{2 h}$. Then there exists a q-ary $\left[\left[\frac{q^{2}-1}{2 h}, \frac{q^{2}-1}{2 h}-2 d+3, d ; 1\right]\right]$ EAQMDS, where $\frac{q+1}{2 h}+1 \leq d \leq$ $\frac{(h+1)(q+1)}{2 h}-1$.

Proof: Consider the constacyclic codes over $F_{q^{2}}$ of length $n=\frac{q^{2}-1}{h}$ with defining set $T=\bigcup_{i=\frac{(h-3)(q+1)}{2 h}}^{k}\left\{C_{1+h i}\right\}$, where $\frac{(h-3)(q+1)}{2 h} \leq k \leq q-2, h \in\{3,5,7\}$ and $h \mid(q+1)$ for $q$ be an odd prime power. By Lemma 3.1, there is $c=\left|T_{s s}\right|=1$ if $\frac{(h-1)(q+1)}{2 h}-1 \leq k \leq q-2$. Since every $q^{2}$-cyclotomic coset has one element which must be odd number, we can obtain that $T$ consists of $\left(i-\frac{(h-3)(q+1)}{2 h}+1\right)$ integers $\left\{1+\left(\frac{(h-3)(q+1)}{2 h}\right) h, 1+\left(\frac{(h-3)(q+1)}{2 h}+1\right) h, 1+\left(\frac{(h-3)(q+1)}{2 h}+2\right) h \cdots, 1+k h\right\}$. It implies that $\mathcal{C}$ has minimum distance at least $\left(i-\frac{(h-3)(q+1)}{2 h}+2\right)$. Hence, $\mathcal{C}$ is a $q^{2}$-ary constacyclic code with parameters $\left[n, n-2\left(i-\frac{(h-3)(q+1)}{2 h}+1\right)+1, \geq\left(i-\frac{(h-3)(q+1)}{2 h}+2\right)\right]$. Combining Lemma 2.6 with EA-quantum Singleton bound, we can obtain a EA-quantum MDS code with parameters $\left[\left[\frac{q^{2}-1}{2 h}, \frac{q^{2}-1}{2 h}-2 d+3, d ; 1\right]\right]_{q}$, where $\frac{q+1}{2 h}+1 \leq d \leq \frac{(h+1)(q+1)}{2 h}-1$.(See Table 1 )

Table 1 EAQMDS codes with $n=\frac{q^{2}-1}{2 h}$

\begin{tabular}{llll}
\hline $\mathrm{q}$ & $\mathrm{h}$ & {$[[n, k, d ; 1]]_{q}$} & $\mathrm{~d}$ \\
\hline 11 & 3 & {$[[20,23-2 d, d ; 1]]_{11}$} & $3 \leq d \leq 7$ \\
23 & 3 & {$[[88,91-2 d, d ; 1]]_{23}$} & $5 \leq d \leq 15$ \\
19 & 5 & {$[[36,39-2 d, d ; 1]]_{19}$} & $3 \leq d \leq 11$ \\
29 & 5 & {$[[84,87-2 d, d ; 1]]_{29}$} & $4 \leq d \leq 17$ \\
13 & 7 & {$[[12,15-2 d, d ; 1]]_{13}$} & $2 \leq d \leq 7$ \\
41 & 7 & {$[[120,123-2 d, d ; 1]]_{41}$} & $4 \leq d \leq 23$ \\
\hline
\end{tabular}




\subsection{Lenght $n=2 \lambda(q-1)$ with $8 \mid(q+1)$ and $\lambda \mid(q+1)$}

Let $q$ be an odd prime power with $8 \mid(q+1)$. Let $\lambda$ be an odd divisor of $q+1, n=2 \lambda(q-1)$ and $r=\frac{q+1}{2 \lambda}$. Clearly, $q \geq 7$ and $r \geq 4$. Now, we use $\eta$-constacyclic codes over $F_{q^{2}}$ of length $n$ to construct $q$-ary EA-quantum MDS codes of length $n$, where $\eta \in F_{q^{2}}$ is a primitive $r^{\text {th }}$ root of unity.

Let $\mathcal{C}$ be a $\eta$-constacyclic code of length $n$ over $F_{q^{2}}$ with defining set

$$
T=\left\{1+r i \mid-(4 \lambda-1) \leq i \leq \frac{q-1}{2}+2 \lambda-1\right\} .
$$

Since $2 \lambda t=q+1$ and $q \geq 7$, one can obtain that $0<1+r\left(\frac{q-1}{2}+2 \lambda-1\right)<\frac{q^{2}-1}{2}$ and $-\frac{q^{2}-1}{2}<1-r(4 \lambda-1)<0$.

In [18], if the defining set $T=\{1+r i \mid-(2 t-1) \leq i \leq 4 t-2\}$, Chen et al. have constructed quantum MDS codes with parameters $[[2 \lambda(q-1), 2 \lambda(q-1)-2 d+2, d]]_{q}$, where $2 \leq d \leq 6 \lambda-1$. However, it is very hard to enlarge the minimal distance $d$ for this type quantum MDS codes. In this subsection, in order to enlarge the minimal distance, adding few ebit, we construct a new family of a family of new EA-quantum MDS codes.

Lemma 3.3: Let $q$ be an odd prime power with $8 \mid(q+1)$ and $n=2 \lambda(q-1)$ where $\lambda$ is an odd divisor of $q-1$. If $\mathcal{C}$ is a $q^{2}$-ary negacyclic code of length $n$ with define set $T=\left\{1+r i \mid-(4 \lambda-1) \leq i \leq \frac{q-1}{2}+2 \lambda-1\right\}$ and the decomposition of a defining set $T=T_{s s} \bigcup T_{\text {sas }}$, then

(i) $\left(C_{1}, C_{-q}\right)$ and $\left(C_{1+r\left(\frac{q-1}{2}\right)}, C_{1+(r-4)\left(\frac{q-1}{2}\right)+q-3}\right)$ form skew asymmetric cosets pairs, respectively.

(ii)

$$
\left|T_{s s}\right|= \begin{cases}2, & \text { if }-(4 \lambda-1) \leq i \leq \frac{q-1}{2}-1 \\ 4, & \text { if } \frac{q-1}{2} \leq i \leq \frac{q-1}{2}+2 \lambda-1\end{cases}
$$

Proof:(i) It is clear that $\left(C_{1}, C_{-q}\right)$ forms a skew asymmetric cosets pair. Since $r n=$ $r 2 \lambda(q-1)=q^{2}-1,\left(1+r\left(\frac{q-1}{2}\right)\right) q=q+\frac{r}{2}\left(q^{2}-q\right) \equiv 1+\frac{r}{2}(q-1)-2(q-1)+q-3$ $\equiv 1+(r-4)\left(\frac{q-1}{2}\right)+q-3 \bmod \left(q^{2}-1\right)$.

(ii) Let $T=\left\{1+r i \mid-(4 \lambda-1) \leq i \leq \frac{q-1}{2}+2 \lambda-1\right\}$. Since $\left(C_{1}, C_{-q}\right)$ and $\left(C_{1+r\left(\frac{q-1}{2}\right)}\right.$, $\left.C_{1+(r-4)\left(\frac{q-1}{2}\right)+q-3}\right)$ form skew asymmetric cosets pairs, respectively, $T_{s s}$ comprises the set $\left\{C_{1}, C_{-q}, C_{1+r\left(\frac{q-1}{2}\right)}, C_{1+(r-4)\left(\frac{q-1}{2}\right)+q-3}\right\}$ at least. According to the concept about a decomposition of the defining set $T$, one obtain that $T_{s a s}=T \backslash T_{s s}$. In order to testify $\left|T_{s s}\right|=2$ if $-(4 \lambda-1) \leq i \leq \frac{q-1}{2}-1$ and $\left|T_{s s}\right|=4$ if $\frac{q-1}{2} \leq i \leq \frac{q-1}{2}+2 \lambda-1$, from Definition 
2.2 and Lemma 2.3 , we need to testify that there is no skew symmetric cyclotomic coset, and any two cyclotomic coset do not form a skew asymmetric pair in $T_{\text {sas }}$.

Let $I=\left\{1+r i \mid-(4 \lambda-1) \leq i \leq \frac{q-1}{2}+2 \lambda-1\right\} \backslash\left(1,-q, 1+r\left(\frac{q-1}{2}\right), 1+(r-4)\left(\frac{q-1}{2}\right)+q-3\right)$ and $r=\frac{q+1}{2 \lambda}$. Only we need to testy that for $\forall x \in I,-q x(\bmod r n) \notin I$ and $T_{s s}=$ $\left\{C_{1}, C_{-q}, C_{1+r\left(\frac{q-1}{2}\right)}, C_{1+(r-4)\left(\frac{q-1}{2}\right)+q-3}\right\}$. That implies that if $x, y \in I$, from Lemma 2.3, $C_{x}$ is not a skew symmetric cyclotomic coset, and any $C_{x}, C_{y}$ do not form a skew asymmetric pair if and only if $x+y q \not \equiv 0 \bmod r n$.

Divide $I$ into $\left(2+\frac{r}{2}\right)$ parts $I_{1}=[1-r(4 \lambda-1), 1-r(2 \lambda-1)], I_{2}=[1-r 2 \lambda,-1]$, $I_{3}=[1+r, 1+r(2 \lambda-1)], I_{4}=[1+r(2 \lambda), 1+r(4 \lambda-1)], \cdots, I_{\left(2+\frac{r}{2}-1\right)}=\left[1+r\left(\frac{q-1}{2}-\right.\right.$ $\left.2 \lambda), 1+r\left(\frac{q-1}{2}-1\right)\right]$ and $I_{\left(2+\frac{r}{2}\right)}=\left[1+r\left(\frac{q-1}{2}+1\right), 1+r\left(\frac{q-1}{2}+2 \lambda-1\right)\right] . r n=2 r \lambda(q-1)=$ $q^{2}-1$. If $x, y \in I_{1}$, then $-2 n<-2\left(q^{2}-1\right)+(r-3)(q+1)=(1-r(4 \lambda-1))(q+1) \leq$ $x+y q \leq(1-r(2 \lambda-1))(q+1)=-\left(q^{2}-1\right)-(r-1)(q+1)<-n$; If $x, y \in I_{2}$, then $-n<-\left(q^{2}-1\right)+(q+1)=(1-r 2 \lambda)(q+1) \leq x+y q \leq-(q+1)<0 ;$ If $x, y \in I_{3}$, then $q+1<(1+r)(q+1) \leq x+y q \leq(1+r(2 \lambda-1))(q+1)<n$. Using the same method, if $x, y \in I_{\left(2+\frac{r}{2}\right)}$, then $\frac{r}{2} n<\left(1+r\left(\frac{q-1}{2}+1\right)\right)(q+1) \leq x+y q \leq\left(1+r\left(\frac{q-1}{2}+2 \lambda-1\right)\right)(q+1)<$ $\left(\frac{r}{2}+1\right) n$.

Hence, there is no skew symmetric cyclotomic cosets, and any two cyclotomic coset do not form a skew asymmetric pair in $T \backslash\left\{C_{1}, C_{-q}, C_{1+r\left(\frac{q-1}{2}\right)}, C_{1+(r-4)\left(\frac{q-1}{2}\right)+q-3}\right\}$. That implies that $\left|T_{s s}\right|=2$ if $-(4 \lambda-1) \leq i \leq \frac{q-1}{2}-1$ and $\left|T_{s s}\right|=4$ if $\frac{q-1}{2} \leq i \leq \frac{q-1}{2}+2 \lambda-1$.

Theory 3.4: Let $q$ be an odd prime power with $8 \mid(q+1)$ and $n=2 \lambda(q-1)$ where $\lambda$ is an odd divisor of $q-1$. then there exists a q-ary $[[2 \lambda(q+1), 2 \lambda(q+1)-2 d+2 i, d ; 2 i]]$ EAQMDS, where $1 \leq i \leq 2$ and $\frac{q-1}{2}(i-1)+4 \lambda+1 \leq d \leq \frac{q-1}{2}+2(i+1) \lambda$.

Proof: Consider the constacyclic codes over $F_{q^{2}}$ of length $n=2 \lambda(q-1)$ with defining set $T=\bigcup_{i=-(4 \lambda-1)}^{k}\left\{C_{1+r i}\right\}$, where $-(4 \lambda-1) \leq k \leq \frac{q-1}{2}+2 \lambda-1$ and $8 \mid(q+1)$ for $q$ be an odd prime power. By Lemma 3.3, there is $c=\left|T_{s s}\right|=2$ if $-(4 \lambda-1) \leq k \leq \frac{q-1}{2}-1$ and $c=\left|T_{s s}\right|=4$ if $\frac{q-1}{2} \leq k \leq \frac{q-1}{2}+2 \lambda-1$. Since every $q^{2}$-cyclotomic coset has one element which must be odd number, we can obtain that $T$ consists of $k+(4 \lambda-1)+1$ integers $\{1+r(-(4 \lambda-1)), 1+r(-(4 \lambda-1)+1), 1+r(-(4 \lambda-1)+2), \cdots, 1+r k\}$. It implies that $\mathcal{C}$ has minimum distance at least $k+4 \lambda+1$. Combining Lemma 2.6 with EA-quantum Singleton bound, we can obtain a EA-quantum MDS code with parameters $[[2 \lambda(q+1), 2 \lambda(q+1)-2 d+2 i, d ; 2 i]]$ EAQMDS, where $1 \leq i \leq 2$ and $\frac{q-1}{2}(i-1)+4 \lambda+1 \leq$ 
$d \leq \frac{q-1}{2}+2(i+1) \lambda .($ See Table 2$)$

Table 2 EAQMDS codes with $n=2 \lambda(q-1)$

\begin{tabular}{llll}
\hline $\mathrm{q}$ & $\lambda$ & {$[[n, k, d ; c]]_{q}$} & $\mathrm{~d}$ \\
\hline 23 & 3 & {$[[132,136-2 d, d ; 2]]_{23}$} & $13 \leq d \leq 23$ \\
& & {$[[132,138-2 d, d ; 4]]_{23}$} & $24 \leq d \leq 29$ \\
47 & 3 & {$[[276,280-2 d, d ; 2]]_{47}$} & $13 \leq d \leq 35$ \\
& & {$[[276,282-2 d, d ; 4]]_{47}$} & $36 \leq d \leq 41$ \\
79 & 5 & {$[[780,784-2 d, d ; 2]]_{79}$} & $21 \leq d \leq 59$ \\
& & {$[[780,786-2 d, d ; 4]]_{79}$} & $60 \leq d \leq 69$ \\
87 & \multirow{2}{*}{11} & {$[[1892,1896-2 d, d ; 2]]_{87}$} & $45 \leq d \leq 87$ \\
& & {$[[1892,1898-2 d, d ; 4]]_{87}$} & $88 \leq d \leq 109$ \\
103 & 13 & {$[[2652,2656-2 d, d ; 2]]_{103}$} & $53 \leq d \leq 103$ \\
& & {$[[2652,2658-2 d, d ; 4]]_{103}$} & $104 \leq d \leq 129$ \\
\hline
\end{tabular}

\section{SUMMARY}

In this paper, based on classical constacyclic MDS codes with a concept about a decomposition of the defining set of constacyclic codes, we construct two families of $q$-ary entanglement-assisted quantum MDS (EAQMDS) codes by exploiting less pre-shared maximally entangled states. In Table 3, we list the $q$-ary entanglement-assisted quantum MDS codes constructed in this paper. By consuming two pre-shared maximally entangled states, we obtain the EA-quantum MDS codes of $2 \lambda(q-1)$ with the minimal distance upper limit greater than $q$ (odd). These EA-quantum MDS codes are improved the parameters of codes in Ref. [18]. Moreover, consuming only one pair of maximally entangled states, we obtain a family of EA-quantum MDS codes of $\frac{q^{2}-1}{2 h}$ with the minimal distance larger than the standard quantum MDS codes in Ref. [23].

Comparing the parameters with $q$-ary EA-quantum MDS codes, we find that these quantum MDS codes are new in the sense that their parameters are not covered by the codes available in the literature.

Table 3 New parameters of EAQMDS codes 


\begin{tabular}{llll}
\hline Class & length & {$[[n, k, d ; c]]_{q}$} & Distance \\
\hline 1 & $n=\frac{q^{2}-1}{2 h}$ & {$\left[\left[\frac{q^{2}-1}{2 h}, \frac{q^{2}-1}{2 h}-2 d+3, d ; 1\right]\right]_{q}$} & $\frac{q+1}{2 h}+1 \leq d \leq \frac{(h+1)(q+1)}{2 h}-1$ \\
& $h \in\{3,5,7\}$ & & \\
2 & $n=2 \lambda(q-1)$ & {$[[n, n-2 d+2 i, d ; 2 i]]_{q}$} & $\frac{q-1}{2}(i-1)+4 \lambda+1 \leq d \leq \frac{q-1}{2}+$ \\
& $\lambda \mid q-1$ odd, & $1 \leq i \leq 2$ & $2(i+1) \lambda$ \\
& $8 \mid(q+1)$, & & \\
\hline
\end{tabular}

\section{Acknowledgment}

This work is supported by the National Natural Science Foundation of China under Grant No.11801564,the National Key R\&D Program of China under Grant No. 2017YFB0802400, the National Natural Science Foundation of China under grant No. 61373171, 111 Project under grant No.B08038.

\section{References}

[1] A. Calderbank, P. Shor, Good quantum error-correcting codes exist. Phys. Rev. A 54, 1098-1105, 1996.

[2] P. Shor, Scheme for reducing decoherence in quantum memory. Phys. Rev. A 52, 2493C2496, 1995.

[3] A. Calderbank, E. Rains, P. Shor, and N. A. Sloane, Quantum error correction via codes over GF(4), IEEE Trans. Inf. Theory, vol. 44, no. 4, pp. 1369-1387, 1998.

[4] T. Brun, I. Devetak, and M. Hsieh, Correcting quantum errors with entanglement. Science 314, 436-439 (2006)

[5] A. Ketkar, A. Klappenecker, S. Kumar and P. Sarvepalli, Nonbinary stabilizer codes over finite fields. IEEE Trans. Inform. Theory, vol. 52, no. 11, p. 4892-4914, 2006.

[6] M. Hsieh, I. Devetak, and T. Brun, General entanglement-assisted quantum errorcorrecting codes. Phys.Rev, A, 76, 062313, 2007.

[7] M. Wilde, and T. Brun, Optimal entanglement formulas for entanglement-assisted quantum coding. Phys. Rev, A, 77, 064302, 2008. 
[8] C. Lai, and T. Brun, Entanglement Increases the Error-Correcting Ability of Quantum Error-Correcting Codes. Phys. Rev, A, 88, 012320, 2013.

[9] C. Lai, T. Brun, and M. Wilde, Duality in Entanglement-assisted quantum error correction. IEEE Trans Inf. Theory 59, 4020-4024 (2013).

[10] C. Lai, T. Brun and M. Wilde, Dualities and Identities for Entanglement-Assisted Quantum Codes. Quantum Inf. Process, 13:957C990 (2014).

[11] Y. Fujiwara, D. Clark, P. Vandendriessche, M. De Boeck, and V. D. Tonchev, Entanglementassisted quantum low-density parity-check codes, Phys. Rev. A, vol. 82, no. 4, p. $042338,2010$.

[12] M. Hsieh,W. Yen, and L. Hsu, High performance entanglement-assisted quantum LDPC codes need little entanglement, IEEE Trans. Inf. Theory, 57, 1761-1769, 2011.

[13] M. Wilde, M. Hsieh, and Z. Babar, Entanglement-assisted quantum turbo codes, IEEE Trans. Inf. Theory, 60, 1203-1222, 2014.

[14] L. Lu and R. Li, Entanglement-assisted quantum codes constructed from primitive quaternary BCH codes, Int. J. Quantum Inf., 12, 1450015, 2014.

[15] L. Lu, R. Li, L. Guo, Q, Fu, Maximal entanglement entanglement-assisted quantum codes constructed from linear codes, Quantum Inf. Process, 12, 1450015, 2015.

[16] L. Guo and R. Li, Linear Plotkin bound for entanglement-assisted quantum codes. Phys. Rev. A 87, 032309 (2013).

[17] R. Li and Z. Xu, Construction of $[[n, n .4,3]]_{q}$ quantum MDS codes for odd prime power $q$, Phys. Rev. A, 82, 052316-1-052316-4, 2010.

[18] B. Chen, S. Ling, and G. Zhang, Application of constacyclic codes to quantum MDS codes. IEEE Trans. Inform. Theory, 61, 1474-1484, 2015.

[19] X. He, L. Xu, and H. Chen, New q-ary quantum MDS codes with distances bigger than $\frac{q}{2}$. Quantum Inf. Process., 15, 2745-2758, 2016.

[20] L. Jin, H. Kan and J. Wen, Quantum MDS codes with relatively large minimum distance from Hermitian self-orthogonal codes. Des. Codes Cryptogr., 2016, doi:10.1007/s10623-016-0281-9. 
[21] X. Kai, and S. Zhu, New quantum MDS codes from negacyclic codes. IEEE Trans. Inform. Theory, 59, 1193-1197, 2013.

[22] T. Zhang, and Ge, G, Some New Classes of Quantum MDS Codes From Constacyclic Codes. IEEE Trans. Inform. Theory, 61, 5224-5228, 2015.

[23] X. Kai, S. Zhu and P. Li, Constacyclic codes and some new quantum MDS codes. IEEE Trans. Inform. Theory, 60, 2080-2086, 2014.

[24] L. Wang, and S. Zhu, New quantum MDS codes derived from constacyclic codes. Quantum Inf. Process., 14, 881-889, 2015.

[25] Zhang, T. and Ge, G, Quantum MDS code with large minimum distance. Des. Codes Cryptogr., doi:10.1007/s10623-016-0245-0. 2016

[26] R. Li, F. Zuo, Y. Liu, A study of skew asymmetric q2-cyclotomic coset and its application. J. Air Force Eng. Univ. (Nat. Sci. Ed.) 12(1), 87C89, 2011. (in Chinese)

[27] R. Li, F. Zuo, Y. Liu and Z. Xu. Hermitian dual containing BCH codes and Construction of new quantum codes. Quantum Inf. and Comp, 13, 0021-0035, 2013.

[28] F. J. Macwilliams and N. J. A. Sloane, The Theory of Error-Correcting Codes, Amsterdam, the Netherlands: North-Holland, 1977.

[29] W. C. Huffman and V. Pless, Fundamentals of Error-Correcting Codes, Cambridge University Press, Cambridge, 2003.

[30] Y. Yang and W. Cai, On self-dual constacyclic codes over finite fields, Designs, Codes Cryptogr., vol. 74, no. 2, pp. 355-364, 2013.

[31] J. Qian, L. Zhang, Entanglement-assisted quantum codes from arbitrary binary linear codes. Des. Codes Cryptogr., 77(1), 193-202, 2015.

[32] J. Qian, L. Zhang, On MDS linear complementary dual codes and entanglementassisted quantum codes, Des. Codes Cryptogr., 2017, doi:10.1007/s10623-017-0413-x.

[33] J. Chen, Y. Huang, C. Feng, R. Chen, Entanglement-assisted quantum MDS codes constructed from negacyclic codes, Quantum Inf. Process., 2017, doi: 10.1007/s11128017-1750-4. 
[34] L. Lu, R. Li, L. Guo, Y. Ma, Y. Liu, Entanglement-assisted quantum MDS codes from negacyclic codes, Quantum Inf. Process., 2018, doi.org/10.1007/s11128-018-1838-5.

[35] L. Lu, W. Ma, R. Li, Y. Ma, Y. Liu, H. Cao, Entanglement-assisted quantum MDS codes from constacyclic codes with large minimum distance, Finite Fields Appl., 53, 309C325, 2018. 2018.

[36] Galindo, C. Hernando, F. Matsumoto, R.: Diego Ruano Entanglement-assisted quantum error-correcting codes over arbitrary finite fields. Quantum Information Processing (2019), doi.org/10.1007/s11128-019-2234-5.

[37] J. Qian, L. Zhang,: Constructions of new entanglement-assisted quantum MDS and almost MDS codes, Quantum Information Processing (2019) https://doi.10.1007/s11128-019-2197-6.

[38] M. E. Koroglu, Quantum Information Processing (2019) 18: 44. https://doi.org/10.1007/s11128-018-2155-8

[39] X. Chen, Sh. Zhu, X. Kai Entanglement-assisted quantum MDS codes constructed from constacyclic codes Quantum Information Processing, 17(10), 2018, DOI: $10.1007 / \mathrm{s} 11128-018-2044-1$

[40] Y. Liu, R. Li, L. Lv, Y. Ma, Application of constacyclic codes to entanglement-assisted quantum maximum distance separable codes Quantum Information Processing, 17(8), 2018, https://dx.doi.org/10.1007/s11128-018-1978-7

[41] J. Chen, Y. Chen, D. Yu, C. Feng, Y. Huang, R. Chen, Applications of Constacyclic Codes to Some New Entanglement-Assisted Quantum MDS Codes IEEE Access, 7(13), 2019, 136641-136657. 